\title{
A Highly Sensitive In-Situ Turbidity Sensor With Low Power Consumption
}

\author{
Yi HU ${ }^{1,2}$, Lei SUN ${ }^{1,2}$, Shuming $\mathrm{YE}^{1,2^{*}}$, Hang $\mathrm{CHEN}^{1,2}$, \\ Kai JIANG ${ }^{1,2}$, and Jianming PAN ${ }^{3}$ \\ ${ }^{1}$ College of Biomedical Engineering \& Instrumentation Science in Zhejiang University, Hangzhou, 310027, China \\ ${ }^{2}$ Key lab of Biomedical Engineering of Ministry of Education in Zhejiang University, Hangzhou, 310027, China \\ ${ }^{3}$ Laboratory of Marine Ecosystem and Biogeochemistry, State Oceanic Administration (SOA), Hangzhou, 310012, China \\ *Corresponding author: Shuming YE_ E-mail: huyi_zju@163.com
}

\begin{abstract}
A highly sensitive in-situ turbidity sensor with the low power consumption was proposed and evaluated in this study. To meet the practical requirements of the in-situ detection, we have designed the light scattering path, watertight mechanical structure, and ultra-weak scattering light detecting method. Experiments showed that the sensor had a sensitivity of 0.0076 FTU with the concentration range of $0-25 \mathrm{FTU}$ and the R-square of 0.9999 . The sensor could withstand the water pressure in depth of $1000 \mathrm{~m}$ and had the low power consumption in the active mode $10.4 \mathrm{~mA}$, sleep mode $65 \mu \mathrm{A}$ with a supply voltage of $8.4 \mathrm{~V}$. Southern China Sea buoy experiments indicated that the sensor could work well in the actual in-situ environment. In comparison with sensors of other companies, our sensor had relatively more comprehensive performance.
\end{abstract}

Keywords: In-situ turbidity sensor, high sensitivity, low power consumption, water tight mechanical structure

Citation: Yi HU, Lei SUN, Shuming YE, Hang CHEN, Kai JIANG, and Jianming PAN, "A Highly Sensitive In-Situ Turbidity Sensor With Low Power Consumption," Photonic Sensors, 2014, 4(1): 77-85.

\section{Introduction}

Since suspended sediments as ubiquitous water pollutant causes significant environment damage and economic costs, monitoring the sediments concentration in the ocean is attracting increasing attention from the marine ecologist and marine biologist in recent years [1]. Turbidity, a fundamental index of water quality, plays an important role in understanding the physical, geochemical, and biological processes of the marine ecosystem [2-4]. And in-situ turbidity detection with the large temporal and spatial scale is one of the effective techniques to provide high-quality and effective data for scientific research $[5,6]$.
However, the commercial in-situ turbidity sensors with high performance mostly depend on the import in China, such as the sensors from Seapoint Inc., Wetlabs Inc., and Alec Inc. Their high expense makes large temporal and spatial scale marine survey difficult for China. So a native in-situ turbidity sensor with high performance is critical for the development of domestic marine scientific research. This paper discusses the design and performance of an in-situ turbidity sensor in detail.

The turbidity is the optical property of a water sample which causes light to be scattered and absorbed rather than transmitted [7]. In-situ turbidity detection is mainly based on infra light scattered

Received:12 November2013. / Revised version: 15 December 2013

(C) The Author(s) 2013. This article is published with open access at Springerlink.com

DOI: $10.1007 / \mathrm{s} 13320-013-0154-\mathrm{z}$

Article type: Review 
from the suspended sediments in the water. The amount of scattered light has linear correlation with the turbidity of the water when the suspended sediments are at the low concentration [8-10]. As the scattered light is very weak and might be mixed with sunlight interference, detecting the ultra-weak scattering light under sunlight interference becomes the key challenge of the sensor.

Besides, there are some other special requirements for the sensor in the marine in-situ measurement. On one hand, as the in-situ survey might be even hundreds of meters under water, the sensor should have the watertight mechanical structure and could withstand the water pressure of $10 \mathrm{MPa}$ at least. On the other hand, due to the limited power supply in the in-situ survey, the power consumption of the sensor is also very critical $[10,11]$.

According to these constrains and solutions, a highly sensitive turbidity sensor with the low power consumption meeting the practical requirements was developed in this work.

\section{Light scattering path and mechanical structure}

The light scattering path is shown in Fig. 1. A high precision light emitting diode (LED) with the central wavelength of $870 \mathrm{~nm}$, spectral bandwidth of $35 \mathrm{~nm}$ is selected as the emitting light source [12]. The particles in the water scatter the emitting light, and the scattered light runs into the receiver. The receiver uses a matching photodiode S1336 (Hamamatsu) with the most sensitive band at the infrared spectrum. In front of the photodiode, a light filter is placed to attenuate the interference of the other light spectrum. Meanwhile, diaphragm 1 and diaphragm 2 are used to limit the light emitting and detecting area through its mechanical structure. The intersection of the two parts is the reaction area. Considering only the single scattering, the scattering angle $\theta$ is from $\theta_{1}$ to $\theta_{2}$ based on the geometric principle [13, 14]. The light path described above could detect the scattering light and attenuate the interference of other light spectrum without introducing reflectance. However, there should be none obstacle in the reaction area, otherwise they may reflect the excitation light into the receiving tube and destroy the detection.

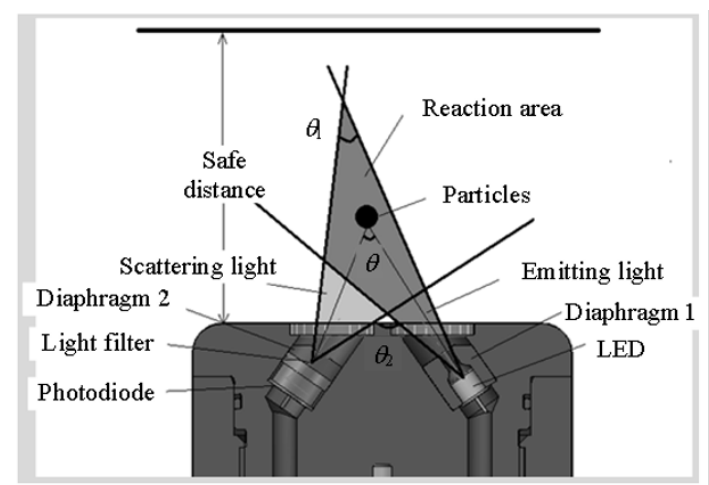

Fig. 1 Light scattering path.

The shape of the sensor is cylindrical as shown in Fig. 2. The scattering light path is in the front part of the sensor, and corundum is selected as the pressure resistant optical window. Stacked circuit boards are installed in the cavity of the watertight structure, and a watertight connector is fixed in the tail of the sensor. The pre-amplification board is placed closely to the photodiode with a shield covering the board to reduce the noise and interference. With the water tight connector, sealing rings, and pressure shell, the sensor could withstand the water pressure of $10 \mathrm{MPa}$ at least.

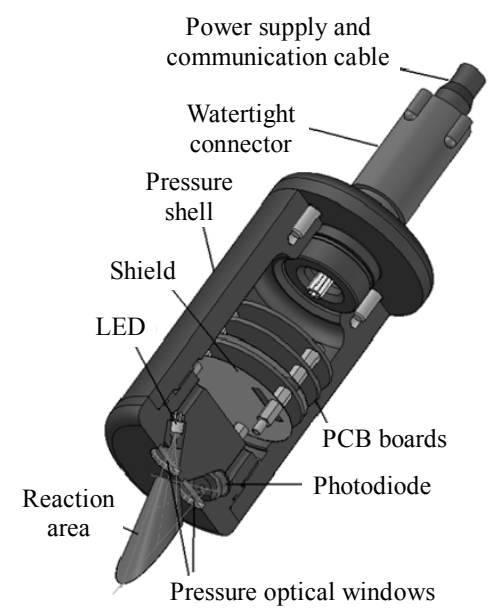

Fig. 2 Mechanical structure of the sensor. 


\section{Ultra-weak light detecting method}

As the sensor has an open light detecting area, sunlight and other background interference could also fall into the light detecting tube. Although the receiving light filter is able to attenuate the interference, it is difficult to extract the scattered light from the rough signal. To overcome this problem, the modulation method is introduced to improve the interference suppression ability of the sensor. This method modulates the emitting light at a high frequency, resulting that the scattered light is at the same frequency band which is different from the interference. Then, the scattered light can be separated from the interference in the frequency spectrum. The schematic diagram is shown in Fig. 3.

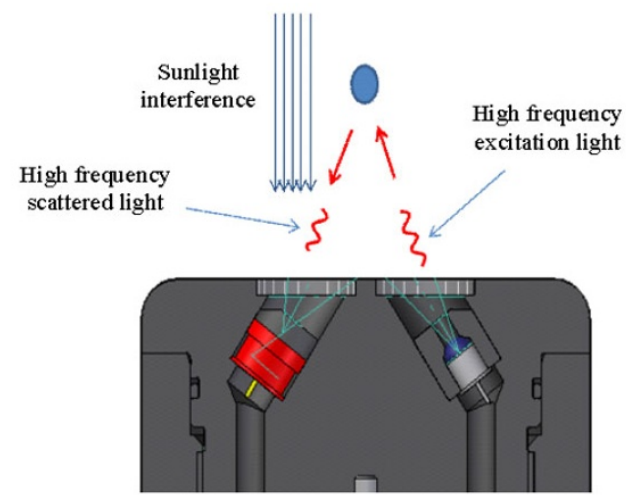

Fig. 3 Single frequency modulation model.

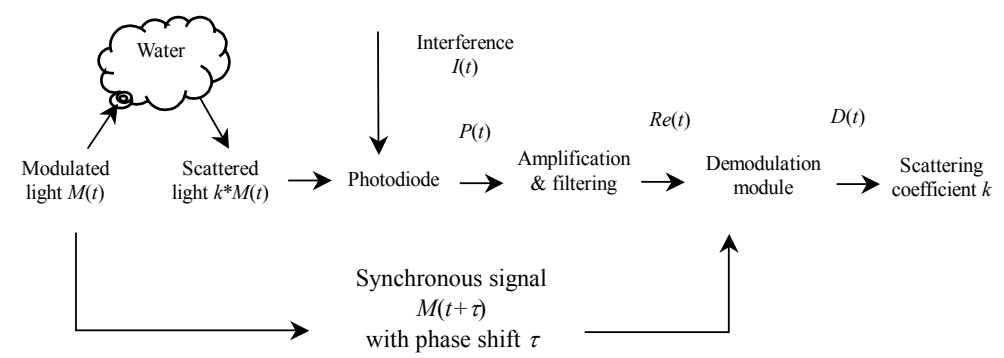

Fig. 4 Mathematical model of the turbidity detection.

The mathematical model of the turbidity detection is shown in Fig. 4, with symbols and significations illustrated in Table 1. In this model, the LED emits the modulated light signal $M(t)$, and particles in the water scatter it. The scattered light signal $k^{*} M(t)$ runs through the light filter and reaches the photodiode; where $k$ is the scattering coefficient which has the linear correlation with the turbidity of the water. Meanwhile, the interference $I(t)$ could also run into the photodiode. The output signal from the photodiode is $P(t)$ as shown in (1):

$$
\begin{gathered}
P(t)=k \times M(t)+I(t) \\
\operatorname{Re}(t)=A \times k \times M(t+\tau)+B \times I(t+\tau)
\end{gathered}
$$

where $A$ is the gain for the useful signal, and $B$ is the gain for the interference.

$$
\begin{aligned}
& D(t)=[A \times k \times M(t+\tau)+B \times I(t+\tau)] \times M(t+\tau) \\
& D(t)=A \times k \times \sin (2 \pi f t+\tau) \times \sin (2 \pi f t+\tau) \\
& +B \times I(t+\tau) \times \sin (2 \pi f t+\tau)
\end{aligned}
$$

Table 1 Symbols and significations in Fig. 4.

\begin{tabular}{cl}
\hline Symbols & \multicolumn{1}{c}{ Significations } \\
\hline$M(t)$ & Modulated light signal \\
$k$ & Scattering coefficient \\
$\tau$ & Phase delay \\
$I(t)$ & Interference signal \\
$M(t+\tau)$ & Synchronous signal \\
$P(t)$ & Received signal from the photodiode \\
$R e(t)$ & $\begin{array}{l}\text { Processed signal after amplification } \\
\text { and filtering }\end{array}$ \\
$D(t)$ & Demodulated signal \\
\hline
\end{tabular}

To avoid the saturation caused by the sunlight interference, a narrow band-pass filter is introduced to attenuate it in the analog front end as shown in Fig. 5. After amplification and filtering, the signal gain $A$, interference gain $B$, phase shift $\tau$ are 
generated to get $\operatorname{Re}(t)$ in (2). Then, $\operatorname{Re}(t)$ is multiplied with the synchronous signal $M(t+\tau)$ in the demodulation part to generate $D(t)$ in (3).

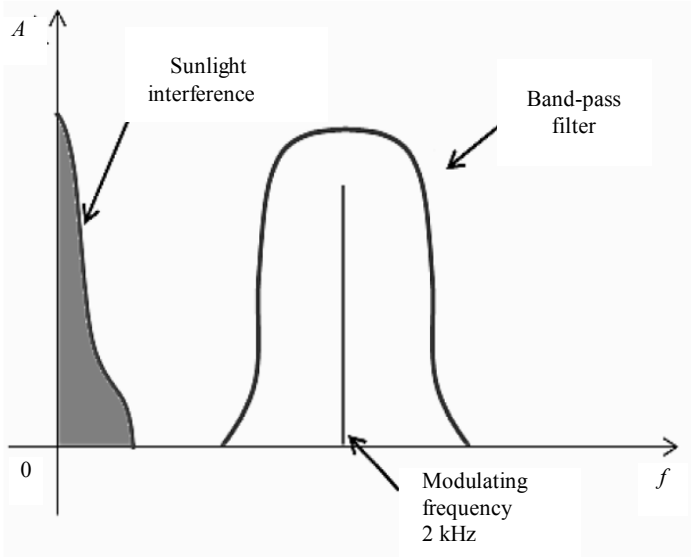

Fig. 5 Narrow band-pass filter to avoid the saturation.

Here, the sine wave $\sin (2 \pi f t)$ is selected as the modulation signal in (4). From the equation, the interference is modulated to a high frequency, and the scattering coefficient $k$ is separated in the form of the direct current (DC). A following ultra-low pass band filter is installed to eliminate the interference, then $A^{*} k / 2$, which has the linear correlation with the turbidity of the water, is left.

Accordingly, we can conclude that this light detecting method could get the scattering coefficient $k$, avoid the saturation caused by the sunlight interference and attenuate it significantly.

\section{Electronics}

Based on the detecting method, the electronics diagram of the system is shown in Fig. 6. The system could be divided into the emitting module and detecting module. The micro controller unit (MCU) generates the $2-\mathrm{kHz}$ square wave as the modulating signal to control the constant current LED driver. L870-40M32 (Epitex) of the narrow light spectrum and small half viewing angle is selected as the LED. All components in the emitting module are designed to improve the stability and purity of the light source [15].

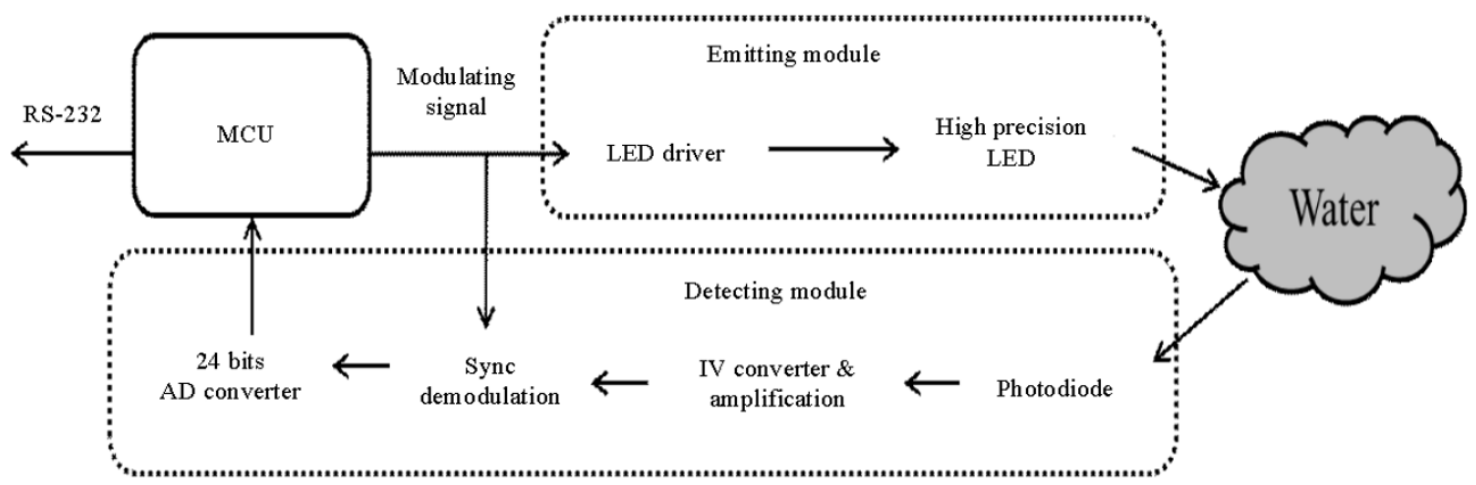

Fig. 6 Electronics diagram of the turbidity sensor.

In the detecting module, a current amplification channel with the high precision and low noise is constructed. A p-n junction (PN) photodiode S1336 (HAMAMASTU) with the high sensitivity and low dark current converts the scattering light into the current signal. A high precision operational amplifier is installed as the I-V converter and narrow band-pass filter to attenuate the sunlight interference (trans-impendence: $1 \mathrm{M} \Omega$, passing band $-3 \mathrm{~dB}$ : $400 \mathrm{~Hz}-6000 \mathrm{~Hz}$ ). In the second stage amplification, a low power amplifier is fixed to boost the signal 50 times and sends the signal to the following sync demodulator. With the sync signal, the demodulator outputs the demodulated signal $D(t)$. After the low pass filter with a cutoff frequency of $1.6 \mathrm{~Hz}$, the DC value containing the scattering coefficient $k$ is separated and sent into a 24-bits ultra-low noise and low power consumption sigma-delta analog to digital (AD) converter. The MCU acquires the result of the conversion through the serial peripheral 
interface (SPI) and calibrates the turbidity value. Then, the value could be stored in an internal flash chip, which has a capacity of 1000,000 data points, or sent out through RS-232 communication port. Meanwhile, an internal real-time clock (RTC) with the back-up battery is installed to decrease the power consumption in the sleep mode of the sensor.

\section{Experiments}

\subsection{Real-time response in the detecting module}

The real-time response in the detecting module is shown in Fig. 7. Waveform $A$ is the sync signal $M(t+\tau)$. Waveform $B$ is the amplified signal of the scattering light, and its amplitude $A^{*} K$ has the linear correlation with the turbidity. Due to the parasitic capacitance in the LED and photodiode, the scattered light signal driven by the $2-\mathrm{kHz}$ square wave looks like a sine wave. After the synchronous demodulation, waveform $\mathrm{C}$, the demodulated signal, is generated. The DC value of the waveform indicates the turbidity of the water. After running through the low-pass filter, the signal is sent into the $\mathrm{AD}$ converter.

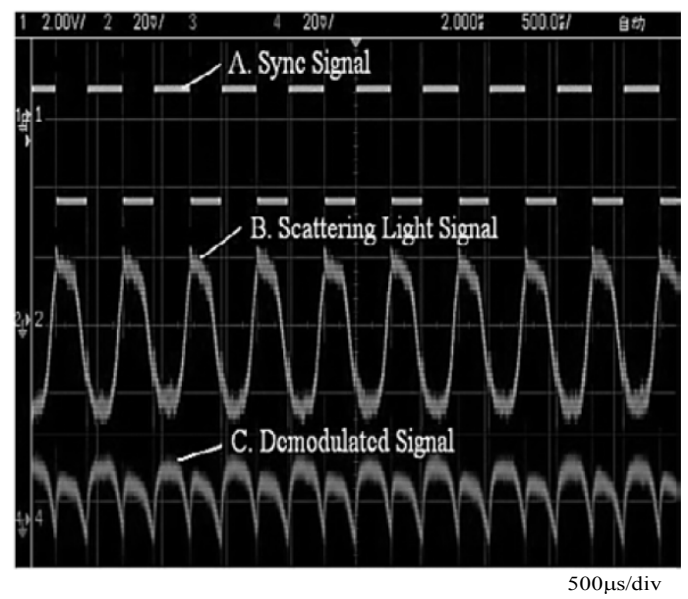

Fig. 7 Real time response in the receiver.

\subsection{Sunlight interference suppression experiment}

In this design, the sunlight interference was suppressed both in the optical part and the electrical part. In the optical part, the scattering light path avoided the direct sunlight radiation, and the receiving light filter attenuated it. In the electrical part, the interference suppression was based on the ultra-weak light detecting method. As constructing stable and quantitative light source is very difficult, we only evaluated the interference suppression ability of the electrical part in this test. The single frequency interference and internal sync signal were injected into the resistors and capacitors (RC) network in Fig. 8, and the output current signal of the network calculated as (5) was used to simulate the output of the photodiode. Then, we recorded the output of the sensor and used different system gains for different inputs to evaluate the sunlight interference suppression ability of the sensor.

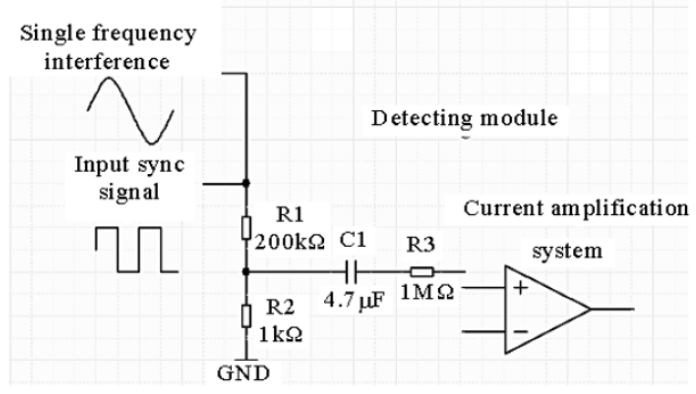

Fig. 8 RC network simulating the output of the photodiode.

$$
I_{\text {out }}=\frac{\left(V_{\text {inf }}+V_{\text {sync }}\right) \times R_{2}}{R_{1}+R_{2}} \div R_{3}
$$

$I_{\text {out }}:$ Output current from the RC network;

$V_{\text {inf }}$ : Input voltage of the interference;

$V_{\text {sync }}$ : Input voltage of the internal sync signal;

$R_{1}, R_{2}, R_{3}$ : Resistors in the network.

The single frequency sine wave ranging from $0.5 \mathrm{~Hz}$ to $20 \mathrm{~Hz}$ with a step of $0.5 \mathrm{~Hz}$ was selected to simulate the low frequency sunlight signal. The internal sync signal was used to simulate the scattered light. We respectively recorded their outputs from the sensor as $R e_{\text {inf }}$ and $R e_{\text {sync }}$, using the signal to interference ratio (STIR) in (6) to 
assess system's interference suppression ability for certain input. As they had the same input amplitude of $3.3 \mathrm{~V}$, we could get (7).

$$
\begin{aligned}
S T I R=-20 & \times \lg \left(\frac{\text { Gain for sync signal }}{\text { Gain for interference }}\right) \\
& =-20 \times \lg \left(\frac{R e_{\text {sync }}}{R e_{\text {inf }}}\right) .
\end{aligned}
$$

In Fig. 9, the STIR values of $0-20 \mathrm{~Hz}$ interference signal were plotted. They were almost all above $60 \mathrm{~dB}$, this means that the gain for the scattered light was 1000 times higher than the gain for the sunlight interference.

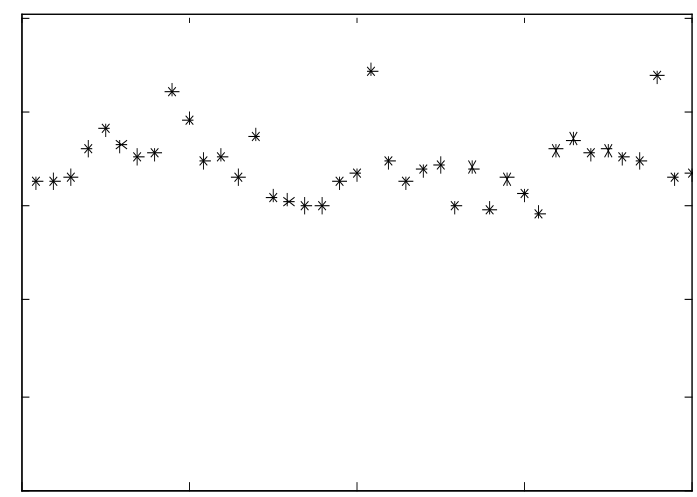

Fig. 9 Signal to interference ratio for $0-20 \mathrm{~Hz}$ input sine wave.

\subsection{Pressure resistant experiment}

The sensor was put into a high pressure kettle as shown in Fig. 10. The kettle could generate $0.1-$ $60 \mathrm{MPa}$ water pressure, and the pressure was added with a step of $0.1 \mathrm{MPa}$. At the pressure of $14 \mathrm{MPa}$, the sensor cracked. So this sensor could work well in depth of $1400 \mathrm{~m}$. We recommended that the sensor should work in depth of $1000 \mathrm{~m}$.

\subsection{Power consumption experiment}

The system could work in two modes, active mode and sleep mode. In the active mode, with a supply voltage of $8.4 \mathrm{~V}$, the sensor consumed $10.6 \mathrm{~mA}$. In the sleep mode, with a supply voltage of $8.4 \mathrm{~V}$, the sensor consumed $65 \mu \mathrm{A}$.

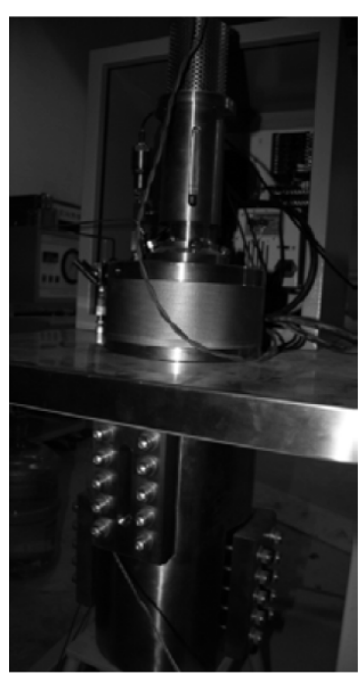

Fig. 10 High pressure kettle.

\subsection{Baseline noise analyses}

The sensor was put into the pure water and opened until it was stabilized. Then, we recorded 100 data points continuously as shown in Fig. 11 and calculated the root mean square (RMS) value to indicate the baseline noise of the sensor. The experiment was carried out in an open environment in the lab with both $50 \mathrm{~Hz}$ interference from the mains and sunlight interference existing. The baseline noise in Fig. 11 was 44.55 .

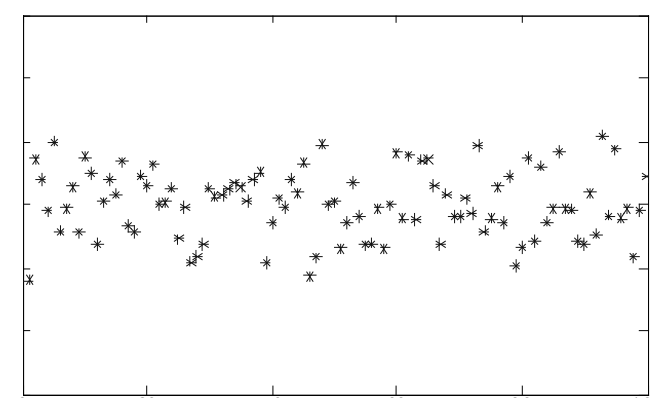

Fig. 11 Baseline noise analysis.

\subsection{Formazine calibration}

We used the standard formazine solution with the concentration of $10000 \mathrm{FTU} / \mathrm{L}$ to calibrate the system in $15 \mathrm{~L}$ pure water and added $1 \mathrm{~mL}$ for eight times, $3 \mathrm{~mL}$ for two times, and $5 \mathrm{~mL}$ for five times. Figure 12 shows the output of the sensor and the 
concentration of Formazine.

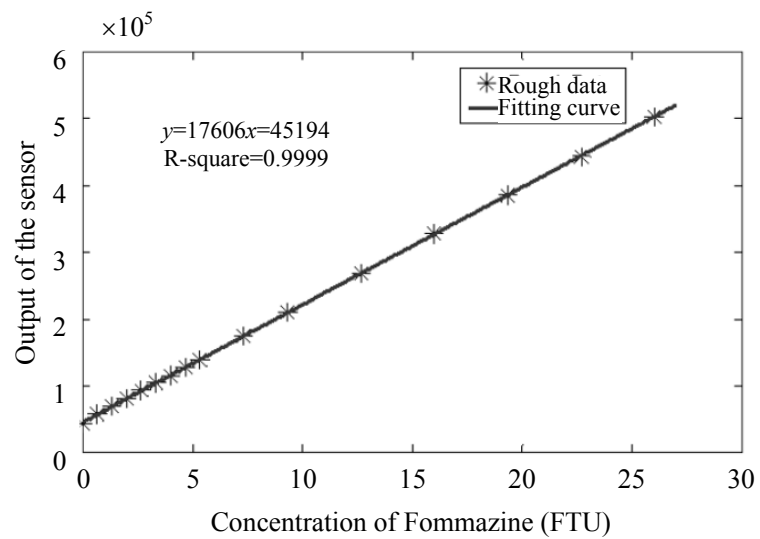

Fig. 12 Standard Formazine calibration for the sensor.

As shown in the figure, the sensor exhibits good linearity in the concentration of $0-25$ FTU with $\mathrm{R}$-square of 0.9999. Assuming that the minimum signal to noise ratio is $3: 1$, we can get the sensitivity $(S)$ with the baseline noise of 44.55 , as calculated in (8):

$$
S=\frac{44.55 \times 3}{17606} \times 1 \mathrm{FTU}=0.0076 \mathrm{FTU} .
$$

And the current resolution (CR) of the amplification system for the photodiode is

$$
C R=\frac{\text { Baseline noise of } \mathrm{AD}}{\mathrm{AD} \text { range }} \times
$$

$$
\text { Reference volatge of } \mathrm{AD}
$$

Trans - impedence of the amplification system

$$
=\frac{44.55 \times 3}{2^{24}} \times \frac{2.5 \mathrm{~V}}{50 \mathrm{M} \Omega}=398.3 \mathrm{fA} .
$$

Table 2 shows the comparison with sensors of

\begin{tabular}{|c|c|c|c|c|}
\hline Company & Seapoint & Wetlabs & ALEC & Our sensor \\
\hline Sensitivity(FTU) & 0.005 & 0.01 & 0.0002 & 0.0076 \\
\hline Supply current(mA) & $\begin{array}{l}6 \mathrm{~mA} \text { (peak); } \\
3.5 \mathrm{~mA}(\text { avg) }\end{array}$ & $\begin{array}{c}60 \mathrm{~mA} \\
\text { (with chlorophyll } \\
\text { detection) }\end{array}$ & $60 \mathrm{~mA}$ & $10.6 \mathrm{~mA}$ \\
\hline Sleep mode & No & $\operatorname{Yes}(150 \mu \mathrm{A})$ & No & $\operatorname{Yes}(65 \mu \mathrm{A})$ \\
\hline Data $\log$ & No & Yes & Yes & Yes \\
\hline
\end{tabular}
other companies, and it indicates that our sensor had relatively more comprehensive performance.

Table 2 Comparison with the sensors of other companies.

\section{Applications}

The sensor was fixed in the buoy located in the Southern China Sea to monitor the in-situ turbidity. The buoy powered the sensor from the solar panels, and the sensor sent the collected data to the central processing unit through RS-232 communication port. Then, the buoy transferred the data to the satellite through the radio signal, and the satellite sent the data back to the receiving antenna on the earth. Finally, the personal computer could get the data through Internet in the form of E-mail. During the monitoring process, the sensor waked up once an hour and got one sample a time. Figure 13 shows the buoy system diagram. Continuous in-situ turbidity data in one week is shown in Fig. 14. From the figure, we can get that although there are some glitches in the curve, the data changes cyclically every day within $0.15 \mathrm{FTU}$.

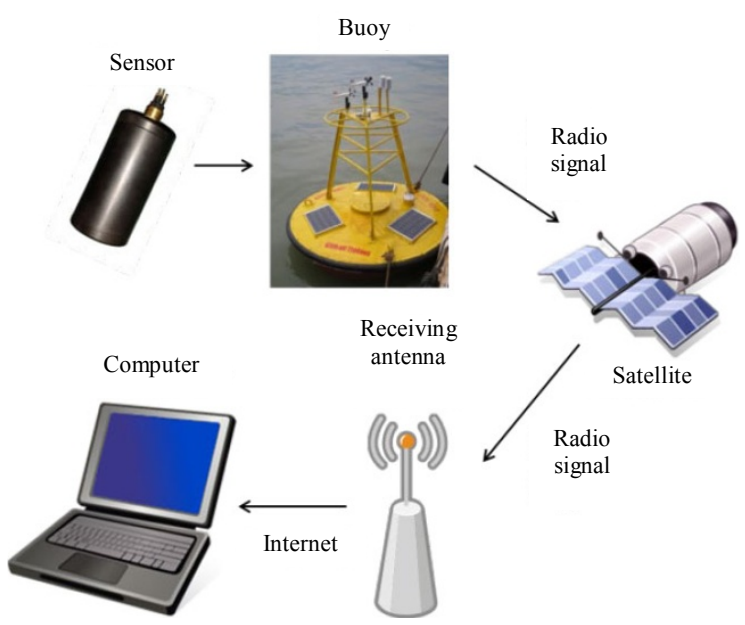

Fig. 13 Buoy System Diagram.

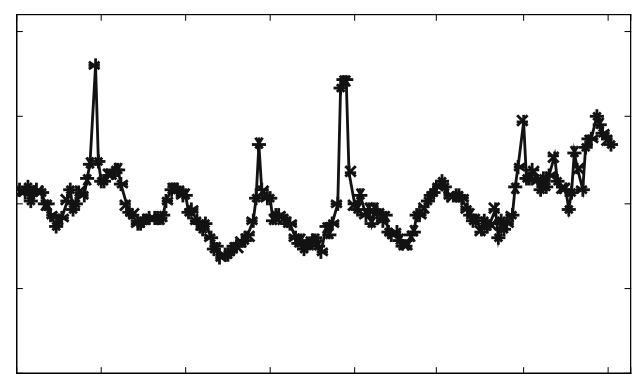

Fig. 14 Southern China Sea one week turbidity data. 


\section{Conclusions}

In this study, a highly sensitive in-situ turbidity sensor with the low power consumption was developed and evaluated. We have designed the light scattering path, the watertight mechanical structure, and the ultra-weak light detecting method to meet the practical requirements of in-situ detecting. The experiments indicated that the sensor had a sensitivity of 0.0076 FTU with the concentration range of $0-25 \mathrm{FTU}$ and the R-square of 0.9999 in the open environment in the lab. The sensor had the low power consumption with the supply current of $10.4 \mathrm{~mA}$ in the active mode and $65 \mu \mathrm{A}$ in the sleep mode. The pressure resistant experiment showed that the sensor could withstand the water pressure in depth of 1000 meters. The long time in-situ experiment indicated that the sensor could meet the practical in-situ detection requirements well in the wild environment. Besides, in comparison with sensors of other companies, our sensor had relatively more comprehensive performance.

\section{Acknowledgment}

This work was supported by 863 National High Technology Research and Development Program in China with contract No. 0032013AA09A411. The authors also would like to thank to the anonymous reviewers.

Open Access This article is distributed under the terms of the Creative Commons Attribution License which permits any use, distribution, and reproduction in any medium, provided the original author(s) and source are credited.

\section{References}

[1] M. Reiter, J. T. Heffner, S. Beech, T. Turner, and R. E. Bilby, "Temporal and spatial turbidity patterns over 30 years in a managed forest of western washington," Journal of the American Water Resources Association, 2009, 45(3):
793-808.

[2] Z. Q. Chen, C. M. Hu, and F. Muller-Karger, "Monitoring turbidity in Tampa Bay using MODIS/Aqua 250-m imagery," Remote Sensing of Environment, 2007, 109(2): 207-220.

[3] W. Shi and M. H. Wang, "Characterization of global ocean turbidity from moderate resolution imaging spectroradiometer ocean color observations," Journal of Geophysical Research-Oceans, 2010, 115(C17): C11022.

[4] R. J. Davies-Colley and D. G. Smith, "Turbidity, suspended sediment, and water clarity: a review," Journal of the American Water Resources Association, 2001, 37(5): 1085-1101.

[5] F. Gohin, "Annual cycles of chlorophyll-a, non-algal suspended particulate matter, and turbidity observed from space and in-situ in coastal waters," Ocean Science, 2011, 7(5): 705-732.

[6] J. V. Loperfido, C. L. Just, A. N. Papanicolaou, and J. L. Schnoor, "In situ sensing to understand diel turbidity cycles, suspended solids, and nutrient transport in clear creek, Iowa," Water Resources Research, 2010, 46(6): W06525.

[7] D. M. Lawler, G. E. Petts, I. D. L. Foster, and S. Harper, "Turbidity dynamics during spring storm events in an urban headwater river system: the upper tame, west midlands, UK," Science of the Total Environment, 2006, 360(1-3): 109-126.

[8] D. M. Lawler and R. M. Brown, "A simple and inexpensive turbidity meter for the estimation of suspended sediment concentrations," Hydrological Processes, 1992, 6(2): 159-168.

[9] T. Erickson, M. S. Division, I. Honeywell, and I. L. Freeport, "Turbidity sensing as a building block for smart appliances," IEEE Industry Application Magazine, 1997, 3(3): 31-36.

[10] S. Mylvaganam and T. Jakobsen, "Turbidity sensor for underwater applications - sensor design and system performance with calibration results," Ocean'98 - Conference Proceedings, 1998, vol. 1-3, pp. $158-161$.

[11] J. F. Orwin and C. C. Smart, "An inexpensive turbidimeter for monitoring suspended sediment," Geomorphology, 2005, 68(1-2): 3-15.

[12] P. K. Dasgupta, I. Y. Eom, K. J. Morris, and J. Z. Li, "Light emitting diode-based detectors absorbance, fluorescence and spectroelectrochemical measurements in a planar flow-through cell," Analytica Chimica Acta, 2003, 500(1-2): 337-364. 
[13] Z. Hong, C. F. Bao, W. F. Qiu, and Z. K. Lu, "On-line turbidity measurement using light surface scattering," Automated Optical Inspection for Industry: Theory, Technology, and Applications Ii, 1998, 3558: 28-30.

[14] K. Ebie, D. Yamaguchi, H. Hoshikawa, and T. Shirozu, "New measurement principle and basic performance of high-sensitivity turbidimeter with two optical systems in series," Water Research, 2006, 40(4): 683-691.

[15] K. F. Ren, F. Xu, X. S. Cai, and J. M. Dorey, "Development of a precise and in situ turbidity measurement system," Chemical Engineering Communications, 2009, 197(2): 250-259. 